\title{
A NEW THERMODYNAMICAL EXPRESSION FOR CALCULATING THE CORRELATION LENGTH
}

\author{
L. DIÓSI and B. LUKÁCS \\ Central Research Institute for Physics, IIungarian Academy of Sciences, \\ P.O. Box 49, H-1525 Budapest 114, Hungary
}

Received 22 April 1985; accepted in revised form 5 August 1985

Starting from recent results on the distribution of the thermodynamic fluctuations we propose a formula which expresses the correlation length by means of the derivatives of thermodynamic potentials.

It was originally revealed by Einstein [1] that equilibrium thermodynamics can provide information on the magnitude of statistical fluctuations in thermodynamical systems. In this paper we shall propose a thermodynamical expression for the correlation length $\xi$ of spatial fluctuations of local thermodynamic characteristics. The correlation length is an important quantity of systems, especially in second order phase transitions [2]. The size $\xi$ may probably characterize the formation of the new phase in first order transitions of classical thermodynamic systems as well as of the dense nuclear matter or the continuum of the very early universe [3].

Let us consider an infinitely large homogeneous equilibrium system characterized by the set of $n$ independent extensive densities $x_{0} \equiv\left(x_{0}^{1}, x_{0}^{2}, \ldots, x_{0}^{n}\right)$. In a given subsystem of volume $V$ the density $x^{i}$ will fluctuate around its average value $x_{0}^{i}$ with the following correlation $[1,4]$ :

$$
\begin{gathered}
\left\langle\left(x^{i}-x_{0}^{i}\right)\left(x^{k}-x_{0}^{k}\right)\right\rangle \sim V^{-1} g^{i k}\left(x_{0}\right) \\
(i, k=1,2, \ldots, n),
\end{gathered}
$$

when $V \rightarrow \infty$. On the $\mathrm{r} h \mathrm{~s}$. the matrix $g^{i k}$ is a pure thermodynamic quantity; it stands for the inverse of the matrix

$g_{i k}(x)=-\partial^{2} s(x) / \partial x^{i} \partial x^{k} \quad(i, k=1,2, \ldots, n)$,

where $s(x)$ is the entropy density function of the given system.
Now, observe that if the homogeneous thermodynamic system in question would be free of spatial correlations then eq. (1) should be correct for arbitrary volumes $V$, not only for $V \rightarrow \infty$. The $1 / V$ factor on the $r$ h.s. of eq. (1) reflects the well-known statistical rule that the squared dispersion of an additive (i.e. extcnsive) quantity must be proportional to the volume if the given system is homogeneous and correlations between different parts of it can be neglected on the actual volume scale.

We shall, therefore, attribute the breaking of eq. (1) to the spatial correlations between the fluctuations of the local thermodynamic characteristics [3].

Recently $[5,6]$, the matrix $g_{i k}$ was applied as the riemannian metric tensor on the space of thermodynamic states of a given system. Onc can expect $[7,8]$ an intimate and direct connection between the metric tensor $g_{i k}$ and the fluctuation probability $p_{V}\left(x \mid x_{0}\right) \mathrm{d}^{n} x$ of the subsystem densities $x^{i}$ ( $V$ is the volume of the subsystem, $x_{0}$ stands for the extensive densities of the large reservoir system).

Let us introduce an evolution variable $\tau=1 / V$ (the underlying physical picture can be found e.g. in ref. [8]). Then the function $p\left(\tau, x \mid x_{0}\right)=p_{V}\left(x \mid x_{0}\right)$ fulfils the following evolution equation [8]

$\frac{\partial}{\partial \tau} p\left(\tau, x \mid x_{0}\right)=\frac{\partial^{2}}{\partial x^{i} \partial x^{k}} g^{i k}(x) p\left(\tau, x \mid x_{0}\right)$,

with summation over the repeated indices $i$ and $k$, according to Einstein's notation. Starting from the 
obvious initial condition [8]

$p\left(0, x \mid x_{0}\right)=\delta^{(n)}\left(x-x_{0}\right)$,

the fluctuation probability is uniquely determined for arbitrary finite equilibrium systems.

Now, we are going to apply eqs. (3), (4) for calculating the correlations

$$
\begin{aligned}
& \left\langle\left(x^{i}-x_{0}^{i}\right)\left(x^{k}-x_{0}^{k}\right)\right\rangle \\
& \equiv \int\left(x^{i}-x_{0}^{i}\right)\left(x^{k}-x_{0}^{k}\right) p\left(\tau, x \mid x_{0}\right) \mathrm{d}{ }^{n} x
\end{aligned}
$$

of the extensive densities in a system of volume $V=$ $1 / \tau$. Assume that the correlation can be expanded into a Taylor series around $\tau=0$. We can evaluate the r h..s. of ey. (5) by substituling the $\tau$-derivatives via eq. (3) and after partial integrations we apply the initial condition (4). Then the result is as follows:

$$
\begin{aligned}
& \left\langle\left(x^{i}-x_{0}^{i}\right)\left(x^{k}-x_{0}^{k}\right)\right\rangle \\
& \quad=g^{i k}\left(x_{0}\right)+\frac{1}{2} \tau^{2} g^{i k}\left(x_{0}\right)_{r s} g^{r s}\left(x_{0}\right)+\mathrm{O}\left(\tau^{3}\right),
\end{aligned}
$$

where the subscripts preceeded by a comma stand for partial derivations. Thus we get the asymptotic correction term to the $r$ h.s. of eq. (1). It is obvious from eq. (6) that the correction term is negligible if $V(=1 / \tau)$ is large enough but it becomes essential that and below a certain correlation volume $V=V_{\text {cor }}$, which must be of the order of $\xi^{d}$ ( $\xi$ is the usual correlation length, $d$ denotes the number of the spatial dimensions of the system).

Stopping at the quadratic term in eq. (6), one can evaluate the criterion for $V_{\text {cor }}\left(\approx \xi^{d}\right)$ by introducing the eigenvectors of the matrix

$M^{i k}=g^{i k}{ }_{r s} g^{r s}$.

Namely, let $v_{a}^{i}$ fulfill the relations

$M^{i r} v_{a r}=m_{a} v_{a}^{i}, \quad g^{r s} v_{a r} v_{a s}=1 \quad(a=1,2, \ldots, n)$.

(The raising of the indices is done, of course, by the metric $g_{i k}$.) Then, in the usual way, one gets that the eigenvectors belonging to different eigenvalues $m_{a}$ are orthogonal to each other. Here we restrict ourselves to the generic case when $M^{i k}$ possesses $n$ eigenvectors. Rewriting the r.h.s. of eq. (6) by means of $m_{a}$ and $v_{a}^{i}$, one gets

$$
\begin{aligned}
& \left\langle\left(x^{i}-x_{0}^{i}\right)\left(x^{k}-x_{0}^{k}\right)\right\rangle \\
& =\sum_{a}\left(1+\frac{1}{2} V^{-1} m_{a}\right) v_{a}^{i} v_{a}^{k} V^{-1}+\mathrm{O}\left(V^{-3}\right) .
\end{aligned}
$$

Therefore the first term dominates if $1 \gg \frac{1}{2} V^{-1} m_{a}$. Eq. (9) clearly indicates that there may exist different correlation mechanisms with different characteristic volumes (lengths). One can choose the maximal of them, and then we have [3]

$V_{\mathrm{cor}}\left(\approx \xi^{d}\right)=\frac{1}{2} \max _{a}\left(m_{a}\right)$.

This is our central result and it should be considered as a possible alternative to Ruppeiner's suggestion in ref. [7]. His suggestion was $V_{\text {cor }}=|R| / 2$, where $R$ is the Ricci scalar curvature of the metric $g_{i k}$. However, this is only a lower bound for $V_{\text {cor }}$. We do not have to perform lengthy calculations for verifying this statement, rather a simple gedankenexperiment will serve. Consider a system whose local state is completely determined by its energy density $\epsilon$. Now, such a system still may contain interactions between particles the system is made of (cf. e.g. the charge-symmetric state of the hot nuclear matter in ref. [9]), then spatial correlations will exist. However, the scalar curvature $R$ is zero for any one-dimensional Riemann space, so there Ruppeiner's correlation vanishes.

Now we are going to investigate two special systems, which are sufficiently simple, and do not contain any interactions in the usual sense, the ideal Boltzmann and the photon gases.

For an ideal Boltzmann gas, the entropy densily function is of the form [4]

$s=\frac{3}{2} n \ln \left(\epsilon / \epsilon_{0}\right)-\frac{5}{2} n \ln (n)$.

where $\epsilon_{0}$ is a scale constant, $\epsilon$ is the energy density and $n$ is the particle density. Hence $x=\left(x^{1}, x^{2}\right)=$ $(\epsilon, n)$, and using eqs. (2), (7) one gets

$$
\begin{aligned}
& g^{i k}=\left[\begin{array}{ll}
(5 / 3) \epsilon^{2} / n & \epsilon \\
\epsilon & n
\end{array}\right], \\
& M^{i k}=\left[\begin{array}{ll}
(20 / 9) \epsilon^{2} / n^{2} & 0 \\
0 &
\end{array}\right]
\end{aligned}
$$

Then eq. (10) yields 


$$
V_{\text {cor }}=2 / 3 n \text {. }
$$

The result is simple enough, but cannot be interpreted as a volume in which the local interactions become important, since in our system all interactions have been neglected. However, eq. (13) possesses a clear physical meaning.

Observe that, apart from the factor $2 / 3$, the correlation volume is the specific volume occupied by a single particle in average. At this order of volumes, the indivisibility of particles itself gives spatial correlation.

The second example is the photon gas. Here the only independent extensive density is the energy density $\epsilon$. The equation of state for a pure photon gas is [4]

$$
\epsilon=\frac{1}{30} \pi^{2}(\hbar c)^{-3} T^{4},
$$

on a temperature scale where the Boltzmann constant is unity. Then the entropy density

$s=\frac{4}{3}\left(2 \pi^{2} / 30\right)^{1 / 4}(\hbar c)^{-3 / 4} \epsilon^{3 / 4}$,

whence

$$
\begin{aligned}
g^{\epsilon \epsilon} & =-\left(\mathrm{d}^{2} s / \mathrm{d} \epsilon^{2}\right)^{-1}=4\left(30 / 2 \pi^{2}\right)^{1 / 4}(\hbar c)^{3 / 4} \epsilon^{5 / 4} \\
M^{\epsilon \epsilon} & =\left(\mathrm{d}^{2} g^{\epsilon \epsilon} / \mathrm{d} \epsilon^{2}\right) g^{\epsilon \epsilon} \\
& =5\left(30 / 2 \pi^{2}\right)^{1 / 2}(\hbar c)^{3 / 2} \epsilon^{1 / 2}
\end{aligned}
$$

Hence, eq. (10) leads to

$$
V_{\text {cor }}=\left(75 / 8 \pi^{2}\right)(\hbar c / T)^{3} \text {. }
$$

We can see that quantum properties lead to correlation between local energy density fluctuations. The correlation length is of the order of the dominant wavelength of the radiation, $\approx \hbar c / 3 T$. This result could be verified by statistical calculation of the energy density fluctuation in the photon gas.

Finally, we are going to apply our main formula (10) in the vicinity of critical points [2]. Here we do not perform a detailed analysis, instead, we show only an illustration. Let us assume that the critical exponent $\alpha$ of the specific heat $C$ determines uniquely the critical exponent $\nu$ of the correlation length $\xi$. It can be shown that the above assumption formally leads to the neglection of all independent extensive variables except the energy density $\epsilon$, when applying eqs. (2), (7), (8) and (10):

$$
\begin{aligned}
g_{\epsilon \epsilon} & =-\partial^{2} s / \partial \epsilon^{2}=-V / T^{2} C \sim\left(T-T_{\mathrm{c}}\right)^{\alpha}, \\
g^{\epsilon \epsilon} & =1 / g_{\epsilon \epsilon} \sim\left(T-T_{\mathrm{c}}\right)^{-\alpha}, \\
M^{\epsilon \epsilon} & =g^{\epsilon \epsilon} \partial^{2} g^{\epsilon \epsilon} / \partial \epsilon^{2} \\
\sim & g^{\epsilon \epsilon}\left[\left(T-T_{\mathrm{c}}\right)^{\alpha} \mathrm{d} / \mathrm{d} T\right]^{2}\left(T-T_{\mathrm{c}}\right)^{-\alpha}, \\
V_{\text {cor }} & \approx \xi^{d}=\frac{1}{2} M^{\epsilon \epsilon} / g^{\epsilon \epsilon} \sim\left(T-T_{\mathrm{c}}\right)^{\alpha-2},
\end{aligned}
$$

where $T_{\mathrm{c}}$ is the critical temperature. Since the correlation length $\xi$ diverges as $\left(T-T_{\mathrm{c}}\right)^{-\nu}$, we arrive at the famous rule [2] $\nu d=2-\alpha$. Thus we have verified that our correlation length $(10)$ is probably consistent with the predictions of critical scaling theories [2].

In conclusion, one can state that the thermodynamic limit preserves information on the spatial correlation of thermodynamic fluctuations. Nevertheless, the investigation of less ideal systems should be necessary.

\section{References}

[1] A. Einstein, Ann. Phys. (Leipzig) 33 (1910) 1275.

[2] H.E. Stanley, Introduction to phase transitions and critical phenomena (Clarendon, Oxford, 1971).

[3] L. Diósi and B. Lukács, KFKI-1984-102.

[4] L.D. Landau and E.M. Lifshits, Statistical physics (Pergamon, London, 1958).

[5] G. Ruppeiner, Phys. Rev. A20 (1979) 1608.

[6] L. Diósi et al., Phys. Rev. A29 (1984) 3343.

[7] G. Ruppeiner, Phys. Rev. A27 (1983) 1116; Phys. Rev. Lett. 50 (1983) 287.

[8] L. Diósi and B. Lukács, Phys. Rev. A31 (1985) 3415.

[9] J.I. Walecka, Phys. Lett. 59B (1975) 109. 